\title{
Evaluación de los conocimientos y actitudes hacia la sexualidad de futuros/as docentes de educación primaria
}

\author{
Sexuality evaluation and knowledge of the future teachers of elementary \\ education
}

\author{
Tania Álvarez-Conde, Yolanda Rodríguez-Castro \\ Facultad de Ciencias da Educación, Universidade de Vigo, España
}

\begin{abstract}
Resumen
El objetivo de este estudio consiste en evaluar el nivel de conocimientos y actitudes hacia la sexualidad que tiene los/as futuros/as docentes de Educación Primaria. Para ello, contamos con una muestra de 149 estudiantes. Se les administró un cuestionario con preguntas sociodemográficas, de conocimientos hacia la sexualidad, la Escala de Transfobia, la Escala de Sexismo ambivalente y la escala de actitudes hacia la sexualidad. Los resultados de este estudio evidencian que los/as futuros/as maestros/as de Educación Primaria muestran un nivel de conocimientos de sexualidad muy bajo (80\%). Los y las estudiantes con menor conocimiento de sexualidad presentan actitudes más erotofóbicas, más transfóbicas y mayor sexismo.

Palabras clave: conocimientos, sexualidad, sexismo, transfobia, educación.
\end{abstract}

\begin{abstract}
The objective of this study is to evaluate the level of knowledge and attitudes that have the future teachers of primary education. For this aim we have a sample of 149 students who were administered a questionnaire with sociodemographic questions, knowledge towards sexuality, the scale of transphobia, the scale of ambivalent sexism and the scale of attitudes towards sexuality. The results of this study show that the future teachers have a very low level of knowledge in sexuality $(80 \%)$. Students with less knowledge of sexuality have more erotophobic, more transphobic attitudes and more sexism.

Keywords: Knowledge, Sexuality, Sexism, Trans-phobia, Education.
\end{abstract}

\section{Introducción}

El estudio científico de la sexualidad a lo largo de la historia siempre fue acompañado por grandes sesgos que condicionaron su perspectiva y entendimiento como el sesgo patológico o medicalizado, el sesgo sexista y el sesgo heterosexista (Rodríguez, Lameiras, Carrera \& Alonso, 2014) generando muchos de los comportamientos de riesgo no saludables así como la asunción de actitudes negativas hacia la sexualidad. Estas actitudes se fundamentan en la difusión de mitos dejados a su suerte por la falta de formación, creados en una sociedad patriarcal y selectiva, provocando así consecuencias preocupantes en el colectivo de los/as más jóvenes por falta de formación, orientación y protección como son embarazos no deseados, infecciones de transmisión sexual (ITS), abusos sexuales y violencia de género (Hurtado \& Olvera, 2013) y otros temas preocupantes y en riesgo en el S. XXI como son: el sexting/sextorsión, el grooming, el ciberbullying o ciberdating violence (Alonso \& Rodríguez, en prensa).

Con respeto al concepto de sexualidad este fue empleado por primera vez en el siglo XIX según Foucault (Lameiras, Carrera \& Rodríguez, 2013). Hoy en día contamos con diversas conceptualizaciones sobre la sexualidad cada vez más abarcadoras. La Organización Mundial de la Salud (OMS, 2006) establece que la sexualidad es uno de los aspectos más significativos del ser humano ya que se encuentra presente al largo de toda su vida. Además, concentra factores biológicos, psicológicos, sociales, políticos, económicos, culturales, legales, éticos, históricos, religiosos y espirituales. En esta línea señala que la sexualidad se vive y se expresa a través de pensamientos, deseos, fantasías, actitudes, creencias, valores, papeles sexuales, conductas y relaciones interpersonales. En definitiva, la sexualidad ya no es tan sólo "un instrumento" para la reproducción humana. Sino que evoluciono a un concepto sin ningún tipo de sesgo, ni patologización dando a conocer la diversidad sexual (Lameiras \& Carrera, 2009).

Por otra parte, el término de Educación Sexual está compuesto por la unión de educación y sexualidad. Tal y como manifiestan Lameiras y Carrera (2009) sólo contando con la "arma" de la educación es posible crear sociedades más tolerantes y demócratas, que hagan con la utopía una dimensión crítica y alternativa para seguir evolucionando.

Con respeto a las características definitorias de una adecuada Educación Sexual serían según Lameiras y Carrera (2009): i.) Tratarse de un proceso lento y gradual a lo largo de todo el ciclo vital. ii.) Mantener una perspectiva integradora de la sexualidad. iii.) Ser susceptible a una posición transformadora y crítica sobre la sexualidad. iv.) Ser un derecho de todas las personas. v.) Estar compuesta tanto por conceptos como por actitudes y comportamientos. vi.) Tener por objetivo principal mejorar la calidad de vida de las personas. vii.) 
fundamentarse con el conocimiento de uno y de una incluso, de la propia identidad. viii.) Comprometerse a transmitir actitudes positivas hacia la sexualidad.

Con respeto al estado de la cuestión en relación con los conocimientos y creencias de la sexualidad se afirman que existen grandes deficiencias en Educación Sexual (Bautista-Hernández, 2008; Hurtado \& Olvera, 2013; Rodríguez, Sanabria, Contreras \& Perdomo, 2013) a pesar de que los/as estudiantes crean tener buenos conocimientos (Ruiz, Latorre, Beltrán, Ruiz y Vélez, 2005).

Por otra parte, a las relaciones sexuales y la edad de la primera relación sexual es cada vez más temprana. Las principales razones que aluden a los/as adolescentes para mantener relaciones sexuales son por presión social de sus iguales y por presión de su pareja como una forma de "demostración de amor" (Chávez, Petrzalova \& Zapata, 2009).

Lo que sí está comprobado es que el conocimiento de los métodos anticonceptivos y preventivos es realmente bajo según Lameiras, Carrera, Rodríguez, Ricoy, Faílde y Núñez (2011). En un estudio nacional español se constató que casi un $40 \%$ de las personas no usan ningún tipo de método anticonceptivo. Asimismo, según Guerrero, Guerrero, García-Jiménez y Moreno (2008) existe una gran creencia en mitos relacionados con la sexualidad. Y según Faílde, Lameiras, Núñez y Bimbela (2007) esta falta de protección confiere un mayor riesgo de ITS, así como, embarazos no deseados.

En segundo lugar, las actitudes, concretamente las relacionadas con la homofobia, masturbación y aborto, se observan en una investigación que, más del $48 \%$ de la muestra marca un nivel positivo bajo (Hurtado \& Olvera, 2013). Y, por otra parte, en el estudio de García-Vega, Menéndez, García y Rico (2010) se pasa la escala de Actitudes SOS que puntúa con un $25.9 \%$ alto en erotofilia (actitud positiva ante todo el erótico y sexual) y un $26.7 \%$ en erotofobia (actitud negativa ante la sexualidad). Asimismo, otra de las actitudes negativas más preocupantes de nuestra sociedad es el sexismo y los mitos del amor. Del mismo modo, según otra investigación el $38 \%$ de los/as adolescentes no recibieron ningún tipo de información sobre planificación. Pero, de los que sí lo hicieron, el 52\% no entiende los métodos anticonceptivos y un $11 \%$ expresa dudas (González, 2009).

Por lo tanto, el objetivo de este estudio consiste en evaluar el nivel de conocimientos y actitudes hacia la sexualidad que tiene los/as futuros/as docentes de Educación Primaria. También analilzaremos el nivel de sexismo y sus actitudes hacia la no conformidad de género.

\section{Método}

\section{Participantes}

La investigación se implementó en una muestra de 149 estudiantes de los cuatro cursos del Grado en Educación Primaria del Campus de Ourense. De los cuales el 32.2\% son chicos y el $67.8 \%$ son chicas con una media de edad de 21.69 (DT: 4.7).

\section{Instrumentos}

Para la elaboración cuantitativa de este estudio se diseñó un cuestionario ad hoc con datos sociodemográficos y con las siguientes escalas:

1. Escalas de conocimientos en sexualidad. Las dos primeras escalas son adaptadas de Lameiras et al. (2013); Lameiras, Rodríguez, Ojea y Dopereiro (2004) y de Rodríguez et al. (2014). Con ellas se determina el nivel de conocimientos de Identidad corporal y de género, así como, de Conductas sexuales. La escala está compuesta por 35 ítems: 16 evalúan el nivel de conocimiento en Identidad corporal y de género (ítem 1 a 16) y los otros miden el grado de conocimiento en Conductas sexuales (ítem 17 a 35) con un rango de respuesta tipo Likert que va de 1 (definitivamente no) a 5 (definitivamente sí). Las respuestas serán recodificadas como acierto o error: siendo los ítems 1, 2, 4, 6, 10, 12, 13, 15, 16, 21, 23, 24, $25,26,27,28,29,30,31,32,33,34$ "definitivamente no" la respuesta correcta y el resto de ítems $(3,5,7,8,9,11$, $14,17,18,19,20,22,35)$ "definitivamente sí" la respuesta adecuada. La escala cuenta con una fiabilidad de $\alpha=.64$ en la subescala de conocimientos de Identidad corporal y de género y uno $\alpha=.60$ de conocimientos de Conductas sexuales.

2. Escala Ambivalent Sexism Inventory (ASI) (Glick \& Fiske, 1996). Para la elaboración de esta investigación se empleó la versión española reducida de Rodríguez, Lameiras y Carrera (2009), con la que se evalúan las actitudes ambivalentes (hostiles y benevolentes) hacia las mujeres. La escala está compuesta por 12 ítems: seis evalúan el Sexismo Hostil (SH) (ítems 1 a 6) y los otros seis miden el Sexismo Benevolente (SB) (ítems 7 a 12) con un rango de respuesta tipo Likert que va de 1 (totalmente en desacuerdo) a 6 (totalmente de acuerdo), siendo las puntuaciones más altas las que representan el mayor nivel de sexismo. La escala muestra adecuada fiabilidad, contando con un coeficiente alfa de Cronbach de .88 el SH y de .76 para el SB.

3. Escala Transphobia (Hill \& Willoughby, 2005). En el presente estudio se empleó la subescala de Genderism (Transfobia) validada en España por Carrera-Fernández, Lameiras-Fernández, Rodríguez-Castro y Vallejo-Medina (2013). Esta escala está constituida por seis ítems que evalúa actitudes discriminatorias cara las personas que rompen con el modelo de los dos sexos y de los dos géneros, es decir, heterosexualidade. Se contesta con una escala tipo Likert que va de 1 (Muy en desacuerdo) a 7 (Muy de acuerdo), siendo las puntuaciones más altas las que representan mayores actitudes transfóbicas. Esta subescala los mostró un coeficiente alfa de Cronbach en la subescala de Transfobia de .85 .

4. Escala Sexual Opinion Survey (SOS) (Fisher, Byrne, White \& Kelley, 1988) se empleó la versión reducida de Vallejo-Medina, Granados y Sierra (2013). La nueva versión breve de seis ítems (SOS-6) presenta una estructura unidimensional, y en el presente estudio alcanza una adecuada fiabilidad $(\alpha=.84)$. Se contesta con una escala tipo Likert de que va de 1 (Totalmente desacuerdo) a 7 (Totalmente de acuerdo), en la cual la menor puntuación mayor nivel de actitudes erotofóbicas. 
5. Cuestiones relativas a si tienen una materia para la formación en sexualidad en su titulación y si creen necesario ese tipo de formación.

\section{Procedimiento}

Una vez seleccionadas las escalas para nuestro estudio, se solicitó los permisos oportunos a las docentes del Grado, con el objetivo de proceder a pasarlas a los/as estudiantes universitarios/as de todos los cursos del Grado de Educación Primaria de Ourense. Se les solicitó su colaboración anónima y voluntaria. Posteriormente, una vez recogidos los datos, se insertaron en el programa informático IBM SPSS Stadistics para su procesamiento y análisis.

\section{Resultados}

En primero lugar, detectamos un alto nivel de desconocimiento en sexualidad (80\%). Así, el nivel de conocimientos generales sobre la Identidad corporal y de género que presenta el alumnado encuestado de Educación Primaria es bastante bajo, puesto que prácticamente el $90 \%$ no tiene buenos conocimientos en la temática $\left(\mathrm{Chi}^{2}{ }_{(1)}=10.92 ; \mathrm{p}<.01\right)$. Incluso, más del $70 \%$ de los/as estudiantes no conoce los ciclos de menstruación $\left(\mathrm{Chi}^{2}{ }_{(1)}=12.43 ; \mathrm{p}<.001\right)$.

Por otra parte, con respeto a la anatomía humana podemos afirmar que el $76,3 \%$ de los/as estudiantes no localiza el clítoris $\left(\mathrm{Chi}^{2}{ }_{(1)}=6.65 ; \mathrm{p}<.01\right)$. También existe un alto de conocimiento de los órganos sexuales masculinos. Así, sólo el 10\% de las chicas y el $32.7 \%$ de los chicos saben que el cuerpo fabrica aproximadamente una media de 200 millones de espermatozoides a diario $\left(\mathrm{Chi}^{2}{ }_{(1)}=11.71 ; \mathrm{p}<.001\right)$.

En segundo lugar, un $69 \%$ de los/as estudiantes del Grado en Educación Primaria no tienen conocimientos adecuados en relación a las Conductas sexuales, no detectándose diferencias entre chicos y chicas $\left(\mathrm{Chi}^{2}{ }_{(1)}=14.59 ; \mathrm{p}=.250\right)$. Por otra parte, hay que destacar la concienciación de los/as estudiantes en la no equivalencia de los celos a sentir amor, más del $85 \%$ contestó adecuadamente, no obstante, aún existe casi un $15 \%$ que confunde el sentimiento con una prueba de amor $\left(\mathrm{Chi}^{2}{ }_{(1)}=14.57 ; \mathrm{p}<.001\right)$.

Con respeto a los métodos anticonceptivos hay generalmente más porcentaje de fallos (un 60.90\%) que de aciertos, exceptuando, entre otras, "La marcha atrás es un método anticonceptivo natural ya que al no eyacular dentro de la vagina no se pueden dar embarazos", cuestión en la que todavía así solo acierta un $80 \%$ $\left(\mathrm{Chi}^{2}{ }_{(1)}=4,01 ; \mathrm{p}<.05\right)$. En esta misma línea, resaltar que, más del $50 \%$ los/las nuevas estudiantes de esta investigación aún sostienen que la píldora del día de después es un método anticonceptivo $\left(\mathrm{Chi}^{2}{ }_{(1)}=5.71\right.$; $\mathrm{p}<.05)$.

Sin embargo, no puede quedar sin comentar que ningún chico conoce el espermicida como un método químico anticonceptivo no eficaz $\left(\mathrm{Chi}^{2}{ }_{(1)}=7.05 ; \mathrm{p}<.01\right)$.

Por otra parte, en la cuestión "Como futuro/a docente tienes alguna materia en tu titulación de Educación Sexual?" el 97.2\% no encuentra relación a Educación Sexual con ninguna de sus materias del Plan de Estudios. $\left(\mathrm{Chi}^{2}{ }_{(1)}=.67 ; \mathrm{p}=.412\right)$. Finalmente, en relación a la valoración de la importancia de contar con una materia de Educación Sexual en su titulación nos encontramos con que casi el $78 \%$ de la muestra dictamina de "muy importante" $\left(\mathrm{Chi}^{2}{ }_{(1)}=.58 ; \mathrm{p}=.965\right)$.

A continuación, analizamos las diferentes medias de las escalas en función del género. Podemos advertir que los chicos tienen un nivel superior de sexismo y transfobia que las chicas (ver Tabla 1). Con respeto a la correlación entre las escalas se pode observar una fuerte relación entre lo sexismo benevolente y el sexismo hostil, así como, con la transfobia (ver Tabla 1). La mayor sexismo hostil, mayor sexismo benevolente y mayores actitudes de transfobia. En relación con la erotofilia-erotofobia, a mayor conocimiento de Identidad corporal y de género menor erotofobia $(\mathrm{r}=.21 ; \mathrm{p}<.05)$. También a menor conocimientos de Sexualidad, mayor sexismo benevolente $(\mathrm{r}=.38 ; \mathrm{p}<.01)$, hostil $(\mathrm{r}=.51 ; \mathrm{p}<.01)$ y mayor transfobia (ver Tabla 1). Específicamente se observa a menor Conocimiento de Conductas sexuales, es menor el conocimiento en Identidad corporal y de género $(\mathrm{r}=.59 ; \mathrm{p}<.01)$.

Tabla 1.

Correlación entre las escalas de conocimientos y de actitudes.

\begin{tabular}{|c|c|c|c|c|c|}
\hline & 1 & 2 & 3 & 4 & 5 \\
\hline $\begin{array}{c}\text { Sexismo } \\
\text { Benevolente (1) }\end{array}$ & - & & & & \\
\hline $\begin{array}{c}\text { Sexismo Hostil } \\
\text { (2) }\end{array}$ & $.54 * *$ & - & & & \\
\hline Transfobia (3) & $.38 * *$ & $.51 * *$ & - & & \\
\hline $\operatorname{SOS}(4)$ & .11 & -.07 & .02 & - & \\
\hline $\begin{array}{l}\text { Conocimientos } \\
\text { de Identidad de } \\
\text { corporal y de } \\
\text { género (5) }\end{array}$ & $-.28 * *$ & $-.3 * *$ & $-.26^{* *}$ & $.21^{*}$ & - \\
\hline $\begin{array}{l}\text { Conocimientos } \\
\text { de Conductas } \\
\text { sexuales }\end{array}$ & $-.23 * *$ & $-.41 * *$ & $-.23 * *$ & .12 & $.59 * *$ \\
\hline
\end{tabular}

Nota: ${ }^{*} p<.05<; * * p<.01 ; y * * * p<.001$

Con relación a las correlaciones entre los datos sociodemográficos y las seis escalas utilizadas los chicos presentan mayor nivel de sexismo, tanto benevolente $(\mathrm{r}=-.29 ; \mathrm{p}<.01)$ cómo hostil $(\mathrm{r}=-.27 ; \mathrm{p}<.01)$, que las chicas. Con respecto a religión, cuanto más religioso se considere la persona mayor sexismo benevolente $(r=-.26$; $\mathrm{p}<.01)$ y hostil $(\mathrm{r}=-.30 ; \mathrm{p}<.01)$, así como, mayor transfobia $(\mathrm{r}=-.37 ; \mathrm{p}<.01)$. Por otra parte, el estudio demuestra que el hecho haber mantenido una relación de pareja anteriormente implica poseer un mayor nivel de actitudes positivas hacia la sexualidad $(\mathrm{r}=-.31 ; \mathrm{p}<.01)$. Del mismo modo, las personas que consideran muy importante establecer una materia de Educación Sexual en el plan de estudios refleja menor sexismo benevolente $(\mathrm{r}=.34 ; \mathrm{p}<.01)$, sexismo hostil $(\mathrm{r}=.31 ; \mathrm{p}<.01)$ y transfobia $(\mathrm{r}=.34 ; \mathrm{p}<.01)$.

El modelo de regresión de la escala de Conocimientos en tela de juicio es explicado en un $21 \%$ por las variables: transfobia, sexismo benevolente y tener pareja (ver Tabla 2). De forma que los que más conocimientos tienen sobre Identidad corporal y de género son los que tienen actitudes más positivas hacia no conformidad de género, menor nivel de sexismo benevolente y tener pareja. 
Tabla 2.

Modelo de Regresión para la escala de Conocimientos de Identidad corporal y de género.

\begin{tabular}{lcccc}
\hline & F & $\boldsymbol{\beta}$ & t & R2 \\
\hline Conocimientos sobre & $7.33 * * *$ & & & .21 \\
Id. Corp. y de gén. & & -.31 & $-3.12 * *$ & \\
Transfobia & -.23 & $-2.39 *$ & \\
Sexismo benevolente & -.21 & $-2.15 *$ & \\
Tener pareja & & & \\
\hline Nota: ${ }^{*} p<.05<; * * p<.01 ; y * * * p<.001$ & & &
\end{tabular}

El modelo de regresión de la escala de conocimientos de Conducta sexual es explicado en un $32 \%$ por las variables: transfobia, sexismo hostil y tiempo con pareja (ver Tabla 3), de forma que los que más conocimientos tienen sobre conductas sexuales son los que tienen actitudes más positivas hacia la no conformidad de género, menor nivel de sexismo hostil y llevar más tiempo con pareja.

Tabla 3.

Modelo de Regresión para la escala de Conocimientos de Conductas sexuales.

\begin{tabular}{lcccc}
\hline & F & $\boldsymbol{\beta}$ & $\mathbf{t}$ & $\mathbf{R}^{2}$ \\
\hline Conocimientos de & $12.02 * * *$ & & & .32 \\
Conductas sexuales & & & & \\
Transfobia & -.23 & $-2.24 *$ & \\
Sexismo Hostil & -.36 & $-3.54 * * *$ & \\
Tiempo pareja & .28 & $2.94 * *$ & \\
\hline Nota: ${ }^{*} p<.05<; * * p<.01 ; y * * * p<.001$ & & &
\end{tabular}

\section{Discusión}

Nuestros resultados muestran una realidad alarmante ya que detectamos un alto nivel de desconocimientos sobre sexualidad en los/as futuros/las docentes de Educación Primaria. De forma que un $80 \%$ no tiene conocimientos adecuados en sexualidad. Datos comparables con los arrojados por la investigación de Bautista-Hernández (2008).

El análisis de los conocimientos de sexualidad centrados en la Identidad corporal y de género refleja las fuertes carencias en conocer sus órganos sexuales. En esta línea se sitúan los resultados de Del Rosario y Solís (2014) que afirman el gran desconocimiento de anatomía sexual que tienen los/las jóvenes. En relación al conocimiento relativo a los métodos preventivos $\mathrm{y} / \mathrm{o}$ anticonceptivo también detectamos la gran carencia de formación que presenta la muestra. Con el cual, se adecúan a las conclusiones de Lameiras et al. (2011). Por consiguiente, tal y como afirman Carrera, Lameiras y Rodríguez (2013) es urgente y necesario humanizar el conocimiento sexual desde un punto crítico y creativo con el objetivo de ofrecer herramientas y claves que estimulen el pensamiento creando inquietudes para la mayor adquisición de conocimientos.

Hoy en día, según Rodríguez, Lameiras y Carrera (2015), aún podemos observar tanto el sexismo ambivalente. Sin embargo, también existen actitudes negativas hacia la transexualidad, la homosexualidad y el lesbianismo. Siguiendo el estudio de Labori y Labori
(2008) más de un $71 \%$ de las mujeres sufren aún violencia verbal. En este estudio se evidencia que a mayores conocimientos en sexualidad menores actitudes negativas hacia la mujer y la transfobia. Además observamos que los chicos son más propensos a mostrar mayores actitudes sexistas. Datos en la misma línea de los estudios de Rodríguez et al. (2015), de Rodríguez, Lameiras, Carrera y Faílde (2010), de Rodríguez y Magalhães (2013) y lo de Yubero, Larrañaga y Navarro (2007). De igual modo, las actitudes negativas hacia la transfobia también son más destacadas por los chicos, tal y como resulta en el estudio de adolescentes según Rodríguez-Castro, Lameiras-Fernández, Carrera y Vallejo-Medina (2013).

Por otra parte, destacar que las chicas tienden a tener actitudes más negativas hacia la sexualidad que los chicos, resultados comparables con los de García, Rico y Fernández (2017) en una muestra universitaria de España. Con el cual las propias actitudes negativas aún se encuentran presentes en nuestra sociedad y el profesorado, así como los centros educativos son una de las principales fuentes de transmisión a las nuevas generaciones, de manera inconsciente con creencias y opiniones sexistas que estiman sobrepasar (Sánchez, 2009). Por lo tanto, cada centro educativo debería contar con un/a Educador/a Social que, según Alonso y Rodríguez (en prensa) consideran que es el perfil más capacitado para esta formación. En definitiva, tal y como afirman Alonso y Rodríguez (en prensa), la formación en Educación Sexual es una inversión preventiva de futuro para los chavales y las chavalas.

\section{Referencias}

Alonso, P. \& Rodríguez, Y. (en prensa). Educación Sexual y Educación Social en la Escuela. En A.A. V.V. (Eds.), Educación Social e Escola balance dunha década. Santiago de Compostela: CEESG e Nova Escola Galega.

Bautista-Hernández, L. A. (2008). Conocimientos, actitudes y opiniones de las y los universitarios Oaxaqueños respecto a su sexualidad y la importancia de la Educación Sexual en el nivel de educación superior. Centro regional de Investigación en psicología, 2(1), 71-84.

Carrera, M. V., Lameiras M., \& Rodríguez, Y. (2013). Hacia una Educación Sexual que todavía es posible. Informació Psicológica, 4, 4-14.

Carrera-Fernández, M. V., Lameiras-Fernández, M., Rodríguez-Castro, Y., \& Vallejo-Medina, P. (2013). Bullying Among Spanish Secondary Education Students: The Role of Gender Traits, Sexism, and Homophobia. Journal of Interpersonal Violence, 20(10), 1-26.

Chávez, M., Petrzelová, J., \& Zapata, J. (2009). Actitudes respeto a la sexualidad en estudiantes universitarios. Enseñanza e investigación en psicología, 14(1), 137-151.

Del Rosario, M. A., \& Solís, A. N. (2014). Conocimientos y actitudes sexuales en adolescentes del caserío el Papayo, Castilla-Piura. Revista IN CRESCENDO, 1(2),463-469. 
Faílde, J. M., Lameiras, M., Núñez, A. M., \& Bimbela, J. L. (2007). Conducta sexual segura y hábitos de salud en jóvenes españoles de 14 a 24 años. SUMMA Psicológica UST, 4(2), 21-33.

Fisher, W. A., Byrne D., White, L. A., \& Kelley, K. (1988). Erotophobia-erotophilia as a dimension of personality. The Journal of Sex Research, 25, 123-51.

García, E., Rico, R., \& Fernández P. (2017). Sexo, roles de género $\mathrm{y}$ actitudes sexuales en estudiantes universitarios. Psicothema, 29(2), 178-183

García-Vega, E., Menéndez, E., García, P., \& Rico, R. (2010). Influencia del sexo y del género en el comportamiento sexual de una población adolescente. Psicothema, 22(4), 606-612.

Glick, P., \& Fiske, S. T. (1996). The Ambivalent Sexim Inventory: Differentiating Hostile and Benevolent Sexism. Journal of Personality and Social Psychology, 70(3), 491-512.

González, J. C. (2009). Conocimientos, actitudes y prácticas sobre la Sexualidad en una Población Adolescente Escolar. Revista de Salud Pública, 11(1), 14-26.

Guerrero, M. D., Guerrero, M., García-Jiménez, E., \& Moreno, A. (2008). Conocimiento de los adolescentes sobre salud sexual en tres institutos de educación secundaria valencianos. Revista Pediatría de Atención Primaria, 10(39), 43-56.

Hill, D. B., \& Willoughby, B. L. B. (2005). The Development and Validation of the Genderism and Transphobia Scale. Sex Roles, 53(7), 531-544.

Hurtado, M. T., \& Olvera, J. (2013). Conocimientos y actitudes sobre sexualidad en jóvenes universitarios. Revista electrónica de psicología Iztacala, 16(1), 258-268.

Labori, J. R., \& Labori, D. (2008). Problemas de género que subyacen a los problemas de salud sexual. Atención Primaria, 40(6), 311-318.

Lameiras, M. \& Carrera, M. V. (2008). A Educación Sexual no novo milenio: obstáculos e retos. Revista de Formación e Innovación Educativa Universitaria, 1(2), 24-36.

Lameiras, M., \& Carrera, M. V. (2009). Educación Sexual. De la teoría a la práctica. Madrid: Pirámide.

Lameiras, M., Carrera, M. V., Rodríguez, Y., Ricoy, M. C., Faílde, J. M., \& Núñez, A. (2011). Evaluación diagnóstica sobre el conocimiento y viabilidad de uso del preservativo femenino: un estudio cualitativo. Análisis y Modificación de Conducta, 37(154), 1-15.

Lameiras, M., Rodríguez, Y., Ojea, M., \& Dopereiro, M. (2004). PROGRAMA AGARIMOS. Programa coeducativo de desarrollo psicoafectivo y sexual. Madrid: Ediciones Pirámide.

Organización Mundial de la Salud. (2006). Colaboremos por la salud. Informe de la salud en el mundo. Ginebra: OMS.

Rodríguez, A., Sanabria, G., Contreras, M. E., \& Perdomo, B. (2013). Estrategia educativa sobre promoción en salud sexual y reproductiva para adolescentes y jóvenes universitarios. Revista Cubana de Salud Pública, 39(1), 161-174.

Rodríguez, Y., \& Magalhães, M. J. (2013). El sexismo moderno en estudiantes universitarios/as portugueses/as. Revista Interdisciplinr de Ciencias Sociais e Humanas, 1(2), 113-121.

Rodríguez, Y., Lameiras, M., \& Carrera, M. V. (2015). Amor y Sexismo: una peligrosa relación en los y las adolescentes gallegos/as. Revista de Estudios $e$ Investigación en Psicología y Educación, 2, 2-12

Rodríguez, Y., Lameiras, M., \& Carrera, M.V. (2009). Validación de la versión reducida de las escalas ASI y AMI en una muestra de estudiantes españoles. Psicogente, 12(22), 284-295.

Rodríguez, Y., Lameiras, M., Carrera, M. V., \& Alonso, P. (2014). Sexualidade e saúde: proposta didáctica. Vigo: Universidad de Vigo.

Rodríguez, Y., Lameiras, M., Carrera, M. V., \& Faílde, J. M. (2010). Evaluación de las actitudes sexistas en estudiantes españoles/as de educación secundaria obligatoria. Psychología, 4(1), 11-24.

Rodríguez-Castro, Y., Lameiras-Fernández, M., Carrera, M. V., Vallejo-Medina, P. (2013). Validación de la Escala de Homofobia Moderna en una muestra de adolescentes. Anales de psicología, 29(2), 523-533.

Ruiz, A. M., Latorre, C., Beltrán, J., Ruiz, J., \& Vélez, A. (2005). Conocimientos, actitudes y prácticas en salud sexual y reproductiva en una población universitaria. Revista Sogia, 12(3), 86-93.

Sánchez, P. (2009). Prevención de la violencia contra la mujer. Red Social DIM-EDU.

Serrano, I., Dueñas, J. L., Bermejo, R., Coll, C., Doval, J. L., Lete, I., Martínez-Salmeán, J., \& Parrilla, J. J. (2005). Actividad sexual e información y uso de métodos anticonceptivos en la juventud española: resultados de una encuesta nacional. Progresos de Obstetricia y Ginecología, 48(6), 283-288.

Vallejo-Medina, P., Granados, M. R. \& Sierra, J. C. (2013). Propuesta y validación de una versión breve del Sexual Opinion Survey en población española. Revista Internacional de Andrología, 12(2), 47-54.

Yubero, S., Larrañaga, E., \& Navarro, R. (2007). Roles Sexuales y actitudes para la igualdad. En Cid, X. M., e Peres, A. (Eds.), Educación Social, Animación Sociocultural y desarrollo comunitario (pp. 1147-1157). Ourense: Universidade de Vigo, Universidade de Trás-os-Montes e Alto Douro e SIPS. 